\title{
NOMENCLATURE AND NAME ASSIGNMENT RULES FOR THE APS STORAGE RING
}

\author{
Glenn Decker
}

March 16, 1992

\section{Introduction}

Because the APS accelerators are moving into the fabrication/assembly/installation stage, it is important for consistent naming conventions to be used throughout the project. The intent of this note is to dictate the rules to be adhered to when naming devices in the storage ring. These rules are generic in nature, and shall be applied in principle to the other machines as well. It is essential that every component have a unique and, hopefully, easily recognizable name.

Every ASD and XFD group, except for magnets, must interface with the control system. For this reason all device names were developed keeping in mind their actual function, such as controlling or monitoring some device in the ring. Even though magnets are not directly interfaced to the control system, their power supplies are; therefore, a magnet will have the same name as its associated power supply.

\section{General Naming Rules}

The name of a device is read from left to right and divided into a number of "fields"; fields are separated from one another by "delimiters." Software engineers know immediately what this means but for those who don't, a field is just a piece of a name that can be extracted easily. For example, consider the name S14A:Q1. The first $S$ is a letter field denoting the accelerator or major subsystem. The number 14 denotes the sector number. Because it is a number and not a letter, it is easily recognized ("parsed") by the computer as a separate field distinguishable from the letter fields on either side of it (the $S$ and the $A$ ). The $A$ and $Q$ are two different letter fields and they need a delimiter between them (the colon) to let the computer know that there really are two fields, and not just a single field containing two letters. The colon (:) is the official method for separating fields. A field can have many characters, but at this time the entire device name, including all the fields and delimiters, is limited to 28 characters. Therefore, emphasis should be placed on using short, concise device names. 


\section{DISCLAIMER}

This report was prepared as an account of work sponsored by an agency of the United States Government. Neither the United States Government nor any agency thereof, nor any of their employees, make any warranty, express or implied, or assumes any legal liability or responsibility for the accuracy, completeness, or usefulness of any information, apparatus, product, or process disclosed, or represents that its use would not infringe privately owned rights. Reference herein to any specific commercial product, process, or service by trade name, trademark, manufacturer, or otherwise does not necessarily constitute or imply its endorsement, recommendation, or favoring by the United States Government or any agency thereof. The views and opinions of authors expressed herein do not necessarily state or reflect those of the United States Government or any agency thereof. 


\section{DISCLAIMER}

Portions of this document may be illegible in electronic image products. Images are produced from the best available original document. 
The above example shows a general rule for naming components at APS, namely, as you move from left to right through a name, the level of detail becomes increasingly fine. The name S14A:Q1, for example, indicates Storage Ring, Sector 14, Subsector A, Quadrupole, Number 1, and is the name of both the magnet and power supply associated with that magnet. More detailed information is added on the right. For instance, S14A:Q1CURR might indicate the amount of current being run through the magnet coils.

\section{Storage Ring Magnet and Power Supply Naming Conventions}

Figure 1 shows the official naming conventions for storage ring magnets. The sector begins just upstream of the first $\mathrm{H} / \mathrm{V}$ steering corrector located on Girder 1 and ends at the downstream end of the insertion device. Each sector is divided into two subsectors: A and B. Subsector A includes all components within the sector upstream of, and including, sextupole S4. Similarly, subsector B contains all downstream components within the sector (the beam moves from left to right in Figure 1). The bending magnet source and insertion device source in the same sector belong to the same CAT (Collaborative Access Team). Sector 1 contains the source points for beamlines BM1 and ID1. This means that the injection septum magnet is located near the end of sector 39 (see Figure 2).

The magnets are numbered in sequence according to their distance from the nearest insertion device. Although this means that all magnets in the B subsectors are numbered "backwards," it will make the machine easier to use once it is built because of the mirror symmetry about sextupole $\$ 4$. My apologies to those who are stuck figuring out which magnet goes on which girder and in what order.

There are five kinds of magnets (not counting insertion devices) in the ring; quadrupoles, skew quadrupoles, sextupoles, steering correctors, and dipoles. The steering corrector magnets each have two sets of coils and two power supplies, one each for horizontal and vertical orbit correction. This type of magnet is therefore stuck with two names, for example S14A:H2 and S14A:V2, for the same corrector. $I$ hope putting the $(n)$ in parentheses in Figure 1 doesn't confuse anybody. All I meant was for the items in parentheses to be variable -- (n) really is just a number somewhere between 1 and 40 . Similarly, $(\mathrm{H}, \mathrm{V})$ should be replaced by either $\mathrm{H}$ or $\mathrm{V}$, not both. Incidentally, numbers less than ten will not use a leading zero, e.g. use S7A:Q3, not S07A:Q3. 


\section{Accelerator Girder and Vacuum System Naming Conventions}

Certain pieces of hardware in the storage ring, such as girders, are completely divorced from the control system. When referring to girder assemblies for installation purposes, all that is required is the sector number and one additional number, 1 through 6 . Therefore, the second girder in sector 14 will have the symbol 14-2 spray painted on its side. Similarly, the vacuum chamber sections are referred to by sector number (one through forty) and section number (one through six), again for installation purposes. This numbering is simple, useful for installation and maintenance purposes, and consistent with the magnet sector numbering scheme.

The overall accelerator vacuum system, however, is monitored by the control system, and specific sensors should comply with the principles already set forward. Because the vacuum system has no mirror symmetry, the letters A and B are not useful and will not be required in the name. All storage ring vacuum components monitored or controlled by the APS control system shall be of the form $S(n): X X X$ where again $(n)$ is replaced by the sector number. The symbol XXX is a field or fields giving more detail, e.g. representing a gauge, pump, or thermocouple.

The sector demarcation described earlier is not detailed enough for the vacuum group. For vacuum purposes, the sector begins at the upstream flange of SRVC section 1. In particular, this means that the two gate valves located immediately upstream and downstream of insertion device $n$ will both be located in sector $n$.

\section{Storage Ring RF Systems}

The four storage ring rf systems have the distinction of being located in sectors $35,36,37$, and 38 . Rather than referring to them by their sector numbers, it is preferable to describe them as if systems $1,2,3$, and 4 , with system 1 being furthest upstream. All $\mathrm{rf}$ system subcomponents will be referred to as SRF1:XXX, SRF2:XXX, etc., where XXX describes the subcomponent in more detail. For example, SRF2:FWD might refer to storage ring system 2 forward power.

\section{Photon Beamline Components}

All photon source points are located in subsector $B$, so again the A and B contribute no new information. In order to easily distinguish between bending magnet and insertion device beamlines and still maintain the nomenclature philosophy, all beamline component names shall be of the form $S(n) B M: X X X$ or S(n)ID:XXX, where BM and ID are used in place of $A$ and $B$, and further detail is given by XXX. Recall that SIBM:XXX represents devices located on the first beamline encountered as one goes clockwise around the ring starting from the injection point. 
Because there are vacuum components serving similar functions in both the accelerator vacuum system and the photon beamlines, it is important that the XXX portion of the name for these items be consistent between ASD and XFD. The ASD Controls Group will serve as the unifying entity, ensuring that nomenclature consistency is maintained throughout the facility and that every device has its own unique name.

\section{Exceptions, Special Items}

There will always be items which are impossible to name in a totally consistent way. For example, the power supply driving the pulsed injection bumper magnets will be connected in series and span two adjacent sectors. These will have to be given special names on a case-by-case basis.

Another item requiring special attention from the accelerator physics point of view is the naming of the drift spaces between magnets. The convention is that a drift space will be given the same name as the magnet immediately upstream, followed by the symbol $\mathrm{L}$. 


\section{Figure 1}

\section{Storage Ring Sector and}

Magnet Nomenclature

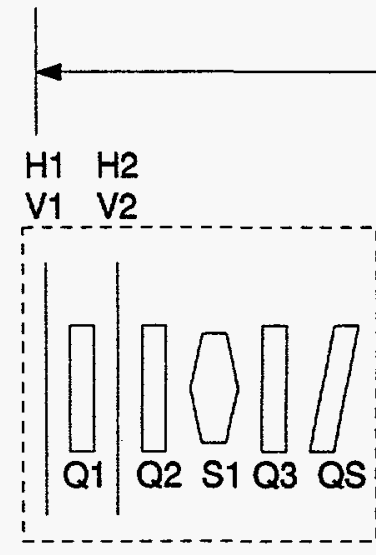

Girder 1

$S(n) A:(H, V) 1$

S(n)A:Q1

S(n)A:P1

S(n)A:(H,V)2

$S(n) A: Q 2$

S(n)A:P2

S(n)A.S1

S(n)A:Q3

S(n)A:QS

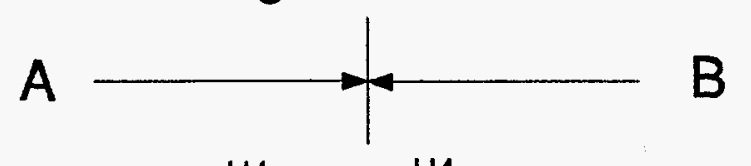

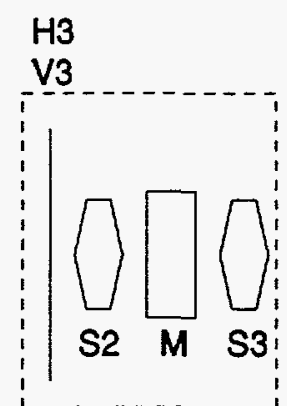

Girder 2

$S(n) A:(H, V) 3$

S(n)A:S2

S(n)A:P3

S(n)A:M

S(n)A:P4

S(n)A:S3

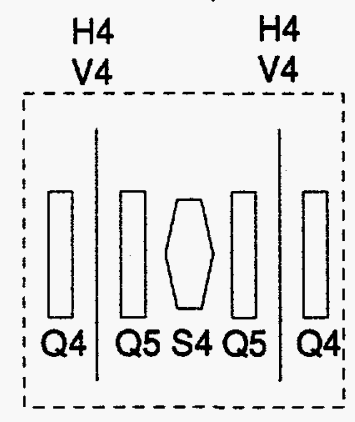

Girder 3

S(n)A:Q4

S(n)A:(H,V)4

S(n)A:Q5

S(n)A:S4

S(n)B:P5

S(n)B:Q5

$S(n) B:(H, V) 4$

S(n)B:Q4

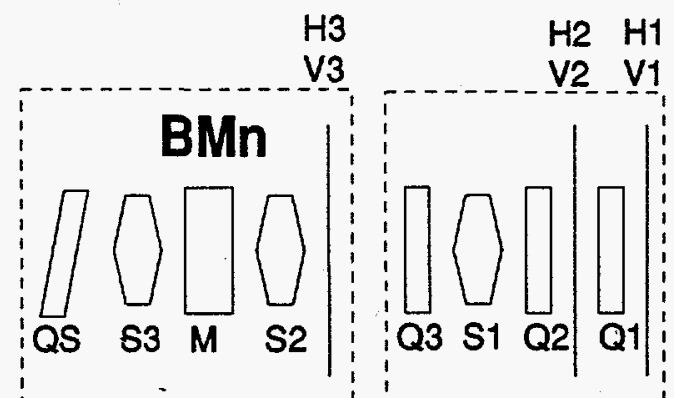

IDn

Girder 4

S(n)B:QS

S(n)B:S3

$S(n) B: P 4$

S(n)B:M

S(n)B:P3

S(n)B:S2

$S(n) B:(H, V) 3$

Girder 5

S(n)B:Q3

S(n)B:S1

$S(n) B: P 2$

$S(n) B: Q 2$

$S(n) B:(H, V) 2$

S(n)B:P1

S(n)B:Q1

$S(n) B:(H, V) 1$

$S=$ Storage Ring

$\mathrm{n}=$ Sector Number

$A, B=$ Upstream, Downstream Half of Sector

$:=$ Delimiter - Do not use period (.)

Q1-Q5 = Quadrupoles

QS = Skew Quadrupole

S1-S4 = Sextupoles

$\mathrm{H} 1-\mathrm{H} 4=$ Horizontal Steering Correctors

V1-V4 $=$ Vertical Steering Correctors

$M=$ Dipoles

$\mathrm{P}=$ Position Monitors

ID = Insertion Device Source

$\mathrm{BM}=$ Bending Magnet Source 


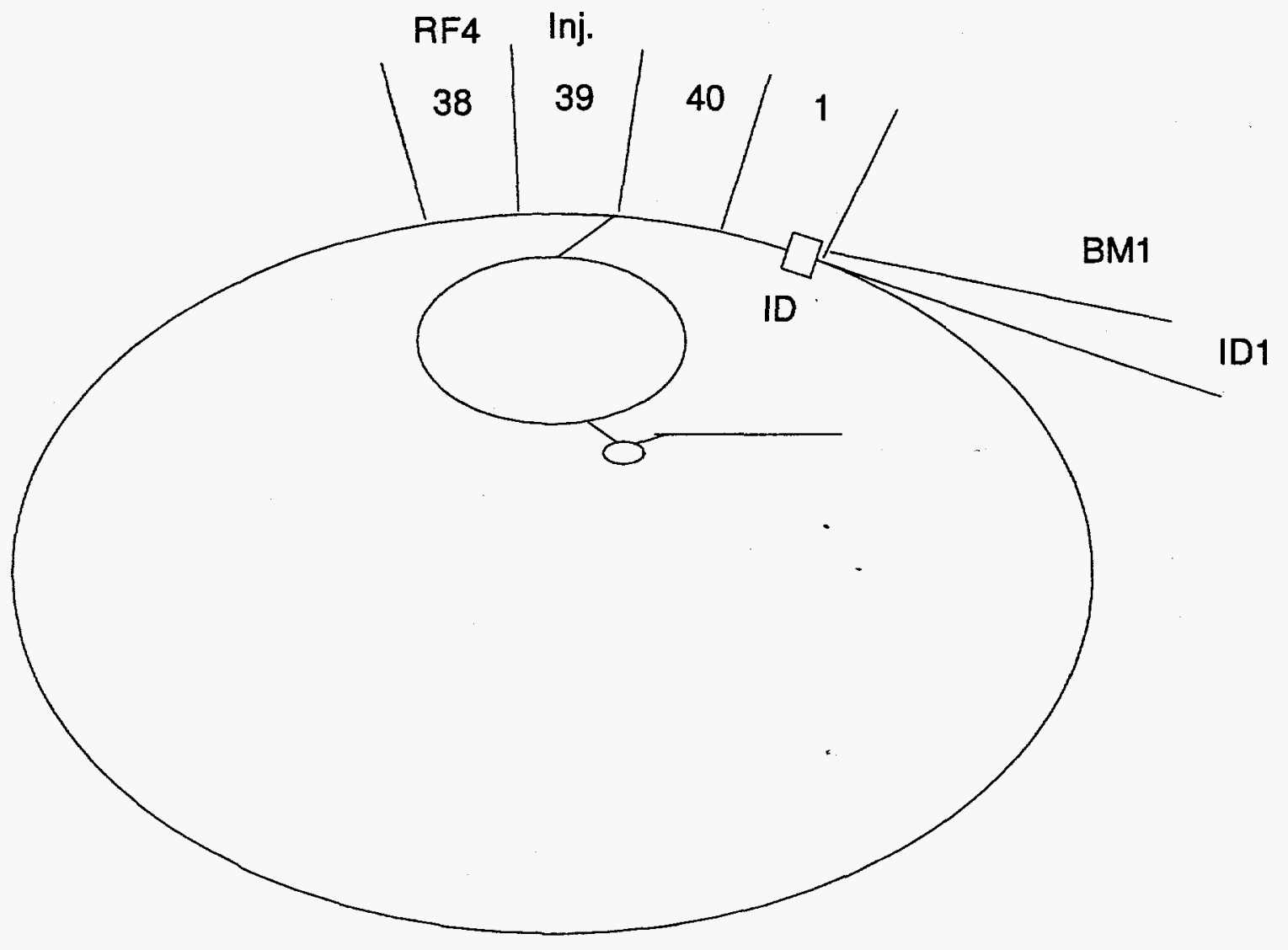

Figure 2

APS Storage Ring

Sector Demarcation 